\title{
Ativação Plaquetária em Formas Clínicas Distintas da Doença Arterial Coronariana (Papel da P-Selectina e de outros Marcadores nas Anginas Estável e Instável)
}

Platelet Activation in Different Clinical Forms of the Coronary Artery Disease (Roll of P-Selectin and others Platelet Markers in the Stable and Unstable Angina)

Margareth L. Venturinelli, André Hovnan, Alexandre de Matos Soeiro, José Carlos Nicolau, José A. F. Ramires, Élbio A. D’Amico, Carlos Vicente Serrano Júnior

Instituto do Coração do Hospital das Clínicas - FMUSP - São Paulo, SP

Objetivo: Os marcadores da ativação plaquetária em geral se apresentam elevados na doença arterial coronariana. Desse modo, procuramos identificar a presença e as potenciais associações de diferentes marcadores da ativação plaquetária.

Métodos: Estudamos pacientes com angina instável $(n=28)$, pacientes com angina estável $(n=36)$ e pacientes sem doença arterial coronariana $(n=30)$; sexo e idade foram estratificados. Os níveis sangüíneos da molécula de adesão P-selectina, do thromboxane B2 e de serotonina foram medidos por imunoensaios enzimáticos.

Resultados: Quando comparamos os grupos, os resultados foram: a P-selectina, o thromboxane B2 e os níveis do serotonina apresentaram-se significativamente mais elevados nos pacientes com angina instável do que nos pacientes com angina estável.

Conclusão: Estes marcadores da ativação plaquetária podem, portanto, identificar formas instáveis de doença arterial coronariana.

Palavras-chave: Ativação plaquetária, doença arterial coronariana.

Objective: Markers of platelet activation are elevated in coronary artery disease. We sought to identify the presence and the potential associations of different markers of platelet activation.

Methods: We studied patients with unstable angina $(n=28)$, patients with stable angina $(n=36)$ and patients without coronary artery disease $(n=30) ;$ sex and age matched. Blood levels of the adhesion molecule P-selectin, Thromboxane B2 and Serotonin were measured by enzyme immunoassays.

Results: When we compared the groups the results were: sP-selectin, thromboxane B2 and serotonin levels were significantly higher in patients with unstable angina than in patients with stable angina.

Conclusion: These markers of platelet activation were able to identify unstable forms of coronary artery disease.

Key words: Platelet activation, coronary artery disease. 


\section{Artigo Original}

A doença arterial coronariana, representada pela aterosclerose, é a doença mais predominante dos dias de hoje, e suas complicações trombóticas são responsáveis por um número altíssimo de mortes e incapacitações. Ao longo dos últimos anos, estudos experimentais e observações clínicas e patológicas proporcionaram uma maior compreensão da formação do trombo e da sua incidência na doença arterial coronariana ${ }^{1}$.

A presença de plaquetas foi identificada no início e na propagação da trombose coronariana ${ }^{2-9}$. As plaquetas formam uma monocamada inicial no local de rompimento da placa aterosclerótica, e diversos ativadores (colágeno, difosfato de adenosina (ADP), epinefrina, serotonina) promovem a ativação plaquetária.

A agregação plaquetária e a formação de trombo decorrentes da ruptura da placa também foram identificadas como importantes mecanismos patogênicos subjacentes à síndrome coronariana de angina instável. A fisiopatologia da angina instável difere da fisiopatologia da angina induzida por esforço, devido ao aumento da demanda de oxigênio do miocárdio. A hipótese de processo patológico na angina instável foi fortemente corroborada pelos resultados de estudos anteriores ${ }^{10-16}$. O processo patológico da angina instável parece envolver a formação temporária de trombo mediada por uma complexa cascata de interações celulares entre o endotélio vascular e as plaquetas na artéria coronária aterosclerótica $^{15}$.

As moléculas de adesão celular desempenham um papel primordial nas interações celulares em diversos processos patológicos, inclusive trombose coronariana, aterosclerose, reestenose após angioplastia coronariana e lesão por reperfusão ${ }^{15,16}$. A P-selectina é um membro da família da selectina e uma glicoproteína de membrana encontrada tanto nos grânulos alfa das plaquetas como nos corpúsculos de Weibel-Palade das células endoteliais. Essas moléculas contêm um domínio N-terminal semelhante à lectina, um domínio semelhante ao fator de crescimento epidérmico, um número variável de repetições de consenso de uma seqüência encontrada nas proteínas reguladoras do sistema complemento, um domínio transmembrana e uma curta cauda citoplasmática $^{16,17}$. Após a ativação celular por agonistas, como a trombina, a P-selectina é rapidamente redistribuída para a superfície celular, ligando-se uma estrutura de carboidrato sializada expressa nos neutrófilos e monócitos por meio de um mecanismo dependente de cálcio semelhante ao da lectina. Assim, a P-selectina medeia as interações entre plaquetas e células endoteliais com leucócitos ${ }^{17-19}$.

Neste estudo, examinamos os níveis plasmáticos de Pselectina solúvel, serotonina e tromboxano $B_{2}$ em pacientes com angina instável e estável para compreender melhor a fisiopatologia dessas síndromes distintas.

\section{Métodos}

Pacientes estudados - A população do estudo consistiu de um grupo de pacientes com angina instável $(n=28)$, um grupo de pacientes com angina estável $(n=36)$ com indicação para revascularização coronariana e um grupo de controle sem DAC ( $n=30)$. Informações clínicas detalhadas, inclusive historia de angina pectoris, fatores de risco para doença arterial coronariana, medicação e intervenções anteriores foram coletadas prospectivamente. Todos os pacientes forneceram um termo de consentimento livre e esclarecido para participar do estudo. Pacientes com infecção, diabete melito, câncer, hepatopatia crônica, insuficiência renal ou doença do tecido conjuntivo, assim como os pacientes que estavam sendo tratados com medicamentos antiinflamatórios ou anticoagulantes (inclusive clopidogrel, aspirina ou inibidores dos receptores da glicoproteína IIb/IIla), foram excluídos do estudo. Os pacientes receberam tratamento farmacológico convencional para angina estável. Todos os pacientes aguardavam cirurgia de revascularização miocárdica sem tomar aspirina, para evitar interação com a ativação plaquetária. Quando havia indicação de anticoagulação com heparina nos casos de angina instável, eram selecionados apenas os pacientes que estavam recebendo heparina de baixo peso molecular, que não afeta a função plaquetária. A tabela 1 apresenta o perfil clínico desses pacientes.

Grupo de angina instável - Os pacientes admitidos no pronto-socorro com angina típica em repouso e eletrocardiograma com supradesnivelamento do segmento ST tinham diagnóstico de angina instável. Do grupo de 48 pacientes submetidos à triagem, 20 pacientes com níveis elevados de enzima CK-MB e troponina I foram excluídos do estudo. Os 25 homens e 3 mulheres restantes tinham estenose $\geq 75 \%$ do diâmetro luminal de pelo menos uma artéria coronária.

Grupo de angina estável - Os 30 homens e 6 mulheres incluídos no grupo de angina estável apresentavam angina de esforço típica, ausência de angina em repouso, teste ergométrico positivo e estenose $\geq 75 \%$ do diâmetro luminal de pelo menos uma artéria coronária.

Grupo de controle - Vinte e três homens e 7 mulheres sem doença arterial coronariana.

Coleta de sangue e dosagem dos marcadores plaquetários - Nos pacientes com angina instável, a coleta de sangue venoso foi feita imediatamente após a entrada no prontosocorro, antes de ser instituída a terapia com anticoagulante. Nos pacientes com angina estável, as amostras de sangue foram coletadas antes da angiografia. Todas as amostras de sangue foram retiradas de uma veia antecubital. As amostras anticoaguladas foram separadas e armazenadas $\mathrm{a}-80^{\circ} \mathrm{C}$ para a dosagem de marcadores plaquetários. As concentrações de sPselectina, serotonina e tromboxano $B_{2}$ no plasma armazenado foram mensuradas com kits de kits de teste imonoenzimático (Bender MedSystems Diagnostics GmbH, Immunotech/Coulter e Amersham Pharmacia, respectivamente).

Análises estatísticas - Os níveis plasmáticos de sP-selectina, serotonina e tromboxano $\mathrm{B}_{2}$ foram comparados por ANOVA unidirecional com o programa de computador SYSTAT 9. Um valor de $\mathrm{P}<0,05$ foi considerado estatisticamente significante.

\section{Resultados}

Níveis plasmáticos de sP-selectina - Os níveis plasmáticos de P-selectina solúvel foram de 274,2 \pm 131,9 ng/mL no grupo de angina instável, 199,7 $\pm 15,78 \mathrm{ng} / \mathrm{mL}$ no grupo de 


\section{Características}

Idade (média \pm S.D)

Homen/mulherMen/women (n)

Dislipidemiaemic ( $\mathrm{n}$ )

TabagismoSmoking

Hipertensãoypertension

Sedentarismo

Obesidadety

História familiar deFamily history of CHD CAD

IM prévioPreviuos MI

História prévia de revascularização e/ou angioplastia coronariana

DAC - doença arterial coronariana; IM - infarto do miocárdio.

$\begin{array}{lll}\begin{array}{l}\text { Angina estável } \\ \mathbf{n = 3 6}\end{array} & \begin{array}{l}\text { Angina instável } \\ \mathbf{n = 2 8}\end{array} & \begin{array}{l}\text { Controle } \\ \mathbf{n}=\mathbf{3 0}\end{array} \\ 56,9 \pm 9,1 & 57,8(12,3 & 56,2(8,7 \\ 30 / 6 & 25 / 3 & 23 / 7 \\ 18 & 14 & 13 \\ 10 & 14 & 8 \\ 14 & 11 & 11 \\ 24 & 25 & 22 \\ 18 & 18 & 15 \\ 10 & 10 & 8 \\ 22 & 8 & 0 \\ 18 & 4 & \end{array}$

Tabela 1- Perfil clínico dos grupos do estudo

angina estável e 176,20 \pm 43,90 no grupo de controle. Esses níveis são apresentados na figura 1. Os níveis plasmáticos de sP-selectina foram significativamente mais elevados no grupo de angina instável do que no grupo de angina estável e no grupo de controle $(p<0,002)$.

Níveis de tromboxano $B 2$ - Os níveis plasmáticos de tromboxano $B_{2}$ foram de $62,89 \pm 14,58 \mathrm{pg} / \mathrm{mL}$ no grupo de angina instável, 21,03 $\pm 6,44 \mathrm{pg} / \mathrm{mL}$ no grupo de angina estável e 19,09 \pm 3,28 no grupo de controle, e são apresentados na figura 2. Os níveis plasmáticos de tromboxano $\mathrm{B}_{2}$ foram significativamente mais elevados no grupo de angina instável do que no grupo de angina estável e no grupo de controle $(p<0,001)$.

Níveis de serotonina - Os níveis plasmáticos de serotonina foram de 2692,61 $\pm 358,42$ nM no grupo de angina instável,

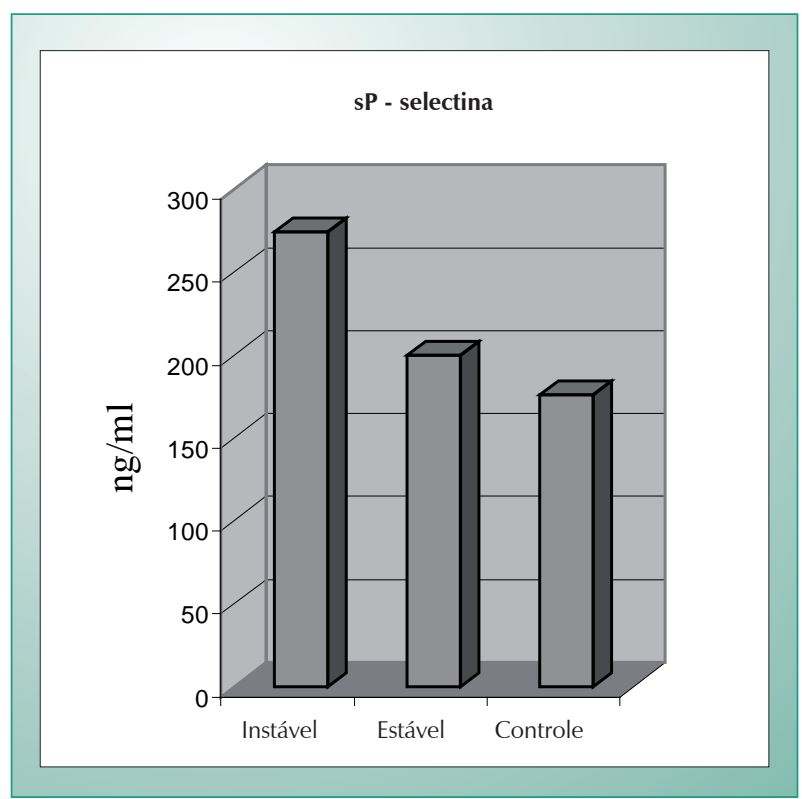

Fig. 1 - níveis de sP-selectina.

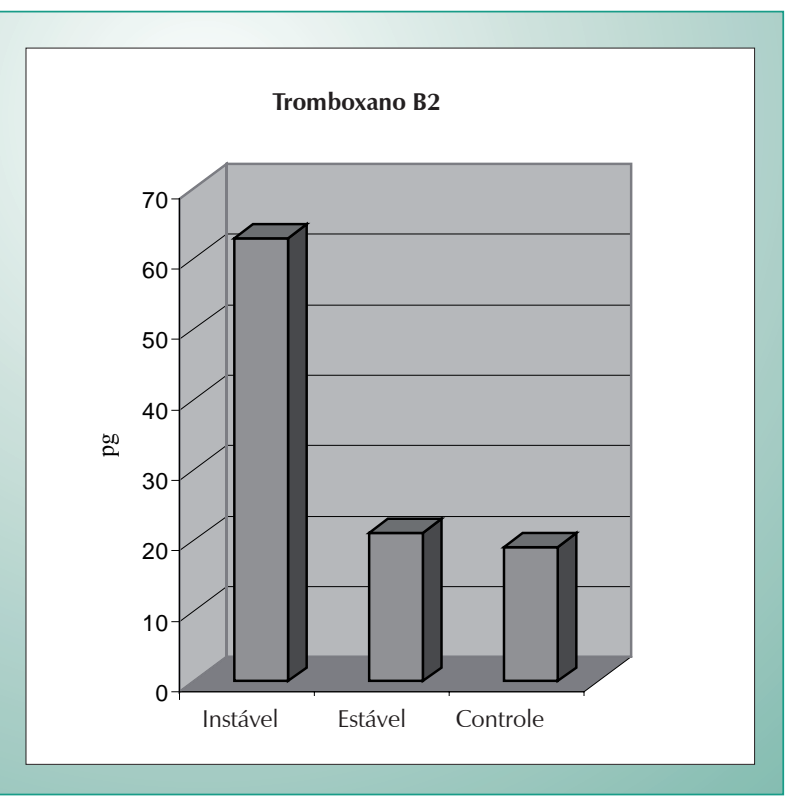

Fig. 2 - Níveis de tromboxano B2.

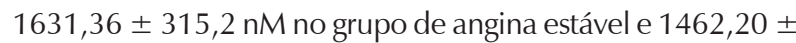
166,36 no grupo de controle, e são apresentados na figura 3. Os níveis plasmáticos de serotonina foram significativamente mais elevados no grupo de angina instável do que no grupo de angina estável e no grupo de controle $(p<0,001)$.

\section{Discussão}

No presente estudo, os níveis plasmáticos de P-selectina, tromboxano $\mathrm{B}_{2}$ e serotonina foram mais elevados em pacientes com angina instável do que nos pacientes com angina estável. Essas observações indicam que a ativação de plaquetas ocorre na circulação durante a angina instável.

P-selectina solúvel - A ruptura da placa coronariana, que resulta na formação de trombo e/ou agregação plaquetária, 


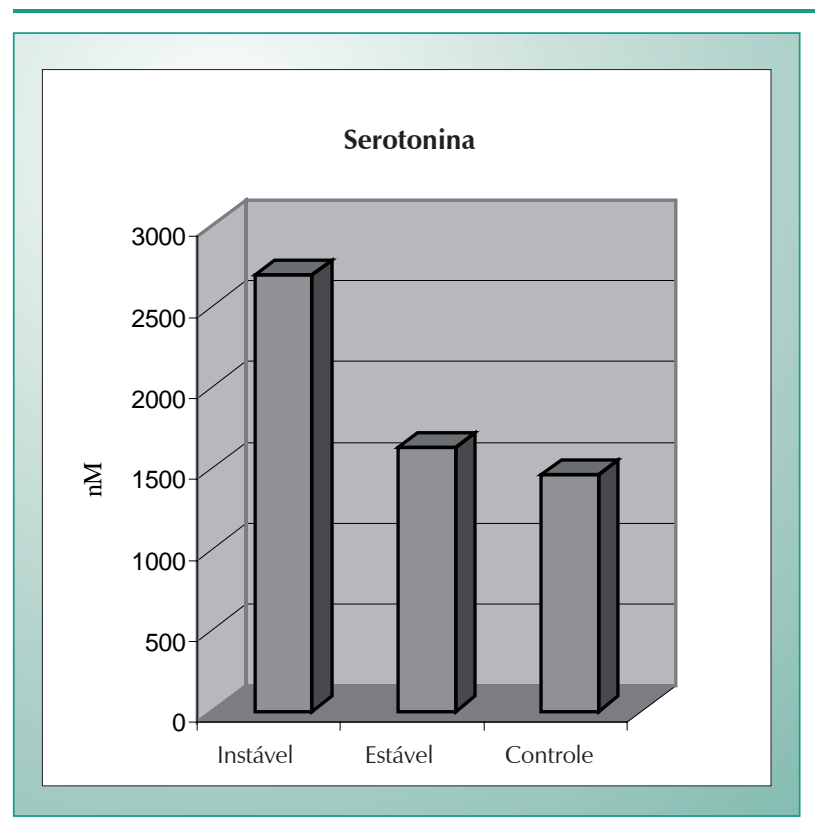

Fig. 3 - Níveis de serotonina.

é apontada como principal mecanismo responsável pelo desenvolvimento de síndromes coronarianas agudas, inclusive angina instável ${ }^{19}$. Estudos recentes ${ }^{19-21}$ mostraram que o aumento dos níveis de sP-selectina em pacientes com angina instável pode ser conseqüência da ruptura da placa e da formação de trombo, bem como de uma interação de plaquetas pelos leucócitos ativados antes da ruptura da placa, e que os níveis de sP-selectina podem ser usados como marcadores de desestabilização da placa na angina instável ${ }^{22}$. No entanto, os níveis de sP-selectina poderiam ser mais elevados nos pacientes com acometimento de múltiplos vasos do que nos pacientes com acometimento de um único vaso ${ }^{23}$. Concluindo, a sP-selectina pode refletir indiretamente o quadro clínico dos pacientes com doença arterial coronariana, com possíveis implicações diagnósticas e terapêuticas ${ }^{2-26}$

Segundo Ault e cols. ${ }^{21}$ existem indícios de ativação plaquetária contínua após evento coronariano isquêmico agudo. A P-selectina associada às plaquetas constitui um marcador sensível de ativação plaquetária; esse marcador

\section{Referências}

1. Napoli C, D'Armiento FP, Mancini FP, Postiglione A, Witztum JL, Palumbo $\mathrm{G}$, et al. Fatty streak formation occurs in human fetal aortas and is greatly enhanced by maternal hypercholesterolemia: intimal accumulation of low density lipoprotein and its oxidation precede monocyte recruitment into early atherosclerotic lesions. J Clin Invest. 1997;100:2680-90.

2. Stary HC, Chandler AB, Glagov S, Guyton JR, Insull W Jr, Rosenfeld ME, et al. A definition of initial, fatty streak, and intermediate lesions of atherosclerosis: a report from the Committee on Vascular Lesions of the Council on Arteriosclerosis, American Heart Association. Circulation. 1994;89:246278 .

3. Ross R. Growth regulatory mechanisms and formation of the lesions of atherosclerosis. Ann NY Acad Sci. 1995;748:1-6.

4. Ross R. The pathogenesis of atherosclerosis: a perspective view for the 1990’s. Nature. 1993;362:801-9. permaneceu elevado por até um mês após a estabilização clínica de pacientes com angina instável ou infarto do miocárdio. A ativação plaquetária persistente pode ser conseqüência de estímulos inflamatórios sustentados ${ }^{20,21}$. Os autores também descobriram uma fraca correlação entre parâmetros de ativação plaquetária e níveis séricos elevados de proteína $C$ reativa ${ }^{21}$.

Tromboxano $B_{2}$ - Os níveis de $\mathrm{TXB}_{2}$ foram estimados pela mensuração de 11-desidrotromboxano B2, o mais abundante metabólito enzimático de TXA2 no plasma. Este estudo demonstrou aumento dos níveis de tromboxano em pacientes com angina instável comparado com pacientes com angina estável. Esses achados são compatíveis com o conceito de que a angina estável reflete o fluxo de sangue restringido através de uma estenose fixa e com a observação de que os inibidores e antagonistas do tromboxano $A_{2}$ não alteram a isquemia miocárdica induzida por exercício ${ }^{27}$. Indicam também que o aumento de tromboxano $B_{2}$ na angina instável pode ser um evento de importância primária, e não apenas secundário à isquemia miocárdica. Além disso, a incidência de infarto do miocárdico e morte é reduzida em 30\% a 50\% nos pacientes com angina instável tratados com aspirina ${ }^{21,27}$.

Serotonina - Estudos clínicos ${ }^{28-30}$ demonstraram que as plaquetas são ativadas e agregadas nos locais de estenose da artéria coronária e de lesão endotelial. As plaquetas ativadas liberam serotonina em quantidades substanciais, causando vasoconstrição e agregação recidivante de plaquetas com reduções cíclicas do fluxo. A serotonina também atua como um fator de crescimento, estimulando a mitogênese e a migração de células da musculatura lisa arterial ${ }^{31}$. Estudos recentes indicam a utilidade da serotonina como um novo marcador de doença vascular aterosclerótica ${ }^{32-34}$.

Este estudo mostra que os níveis de P-selectina, tromboxano $B_{2}$ e serotonina são mais elevados na angina instável do que na angina estável. Portanto, esses marcadores de ativação plaquetária podem nos ajudar a compreender a fisiopatologia da doença arterial coronariana, por refletir indiretamente o quadro clínico de pacientes com doença arterial coronariana, com possíveis implicações diagnósticas e terapêuticas.

\section{Potencial Conflito de Interesses}

Declaro não haver conflitos de interesses pertinentes.

5. Ross R. Atherosclerosis - An inflammatory disease. N Engl J Med. 1999;340:115-26.

6. Azar RR, Waters D. The inflammatory etiology of unstable angina. Am Heart J. 1996;132:1101-6.

7. Serrano Jr. CV, Ramires JA, Venturinelli M, Arie S, D’Amico E, Zweier JC, et al. Coronary angioplasty results in leukocyte and platelet activation with adhesion molecule expression: evidence of inflammatory responses in coronary angioplasty. J Am Coll Cardiol. 1997;29:1276-83.

8. Libby P. Molecular bases of the acute coronary syndromes. Circulation. 1995;91:2844-50.

9. Coller BS. Antiplatelets agents in the prevention and therapy of thrombosis. Annu Rev Med. 1992;43:171-80. 
10. Einsenberg PR. Mechanism of action of heparin and anticoagulant therapy: implications for the prevention of arterial thrombosis and the treatment of mural thrombosis. Coron Art Dis. 1990;1:159-5.

11. Coller BS. Inhibitors of the platelet glycoprotein IIb/IIla receptor as conjunctive therapy for coronary artery thrombolysis. Coron Art Dis. 1992;3:1016-29.

12. Bombeli T, Schwartz BR, Harlan JM. Adhesion of activated platelets to endothelial cells: evidence for a Gpllb/IIla dependent bridging mechanism and novel roles for endothelial intercellular adhesion molecule-1 (ICAM-1), avb3 integrin, and Gplba. J Exp Med. 1998;187:329-39.

13. Handin RI, Loscalzo J. Hemostasis, Thrombosis, Fibrinolysis, and Cardiovascular Disease. In: Braunwald E. Ed. Heart Disease: A Texbook of Cardiovascular Medicine. 4th ed., Philadelphia: WB Saunders, 1990: 1767-89.

14. Coller BS. Diagnostic and therapeutic applications of antiplatelet monoclonal antibodies. Biorheology. 1987;24:649-58.

15. Hawiger J. Formation and regulation of platelet and fibrin hemostatic plug. Human Pathol. 1987;18:111-22.

16. Fitzgerald D, Roy L, Catella F, Fitzgerald G. Platelet activation in unstable coronary disease. N Eng J Med. 1986;315:983-9.

17. Tenaglia AN, Buda AJ, Wilkins RG, Barron MK, Jeffords PR, VOK, et al. Levels of expression of P-selectin, E-selectin, and intercellular adhesion molecule-1 in coronary atherectomy specimens from patients with stable and unstable angina pectoris. Am J Cardiol. 1997;79:742-47.

18. Atalar E, Aytemir K, Haznedaroglu Y, Ozer N, Ovunc K, Aksoyek S, et al. Increased plasma levels of soluble selectins in patients with unstable angina. Int J Cardiol. 2001;78:69-73.

19. Blann AD, Lip GYH. Hypothesis: is soluble P-selectin a new marker of platelet activation? Atherosclerosis. 1997;128:135-138.

20. Atalar E, Haznedaroglu Y, Aytemir K, Ozer N, Aksoyek S, Ovunc K, et al. Circulating adhesion molecules in patients with stable coronary artery disease. Int J Hematol. 2000;72(4):507-11.

21. Ault AK, Cannon CP, Mitchell J, McCahan J, Tracy RP, Novotny WF, et al. Platelet activation in patients after an acute coronary syndrome:results from the TIMI-12 trial. J Am Coll Cardiol. 1999;33(3):634-9.

22. Draz N, Hamdy MS, Gomaa Y, Ramzy AA. Soluble P-selectin is a marker of plaque destabilization in unstable angina. Egypt J Immunol. 2003;10(1):83-7.
23. Fang L, Wei H, Mak KH, Xiong Z, SongJ, Wang D, et al. Markers of low-grade inflammation and soluble cell adhesion molecules in Chinese patients with coronary artery disease. Can J Cardiol. 2004;20(14):1433-8.

24. Guray U, Erbay AR, Guray Y, Yilmaz MB, Boyaci AA, Sasmaz H, et al. Levels of soluble adhesion molecules in various clinical presentations of coronary atherosclerosis. Int J Cardiol. 2004;96(2):235-40.

25. Romuk E, Skrzep-Poloczek B, Wojciechowska C, Tomasik A, Birkner E, Wodniecki J, et al. Selectin-P and interleukin-8 plasma levels in coronary heart disease patients. Eur J Clin Invest. 2002;32(9):657-61.

26. Mizia-Stec K, Mandecki T, Zahorska-Markiewicz B, Janowska J, Szulc A Jastrzebska-Okon K, et al. P-selectin and E-selectin in serum of patients with coronary artery disease. Pol Arch Med Wewn. 2001;106(6):1137-44.

27. Pakala R, Willerson JT, Benedict CR. Effect of serotonin, tromboxane A2, and specific receptor antaonists on vascular smooth muscle cell proliferation. Circulation. 1997;96:2280-86.

28. Seuwen K, Pouyssegur J. Serotonin as a growth factor. Biochem Pharmacol. 1990;39:985-990.

29. Willerson JT, Yao SK, McNatt J, Bebedict CR, Anderson HV, Golino P, et al Frequency and severity of cyclic flow alternations and platelet aggregation predict the severity of neointimal proliferation following experimental coronary stenosis and endothelial injury. Proc Natl Acad Sci USA. $1991 ; 88: 10624-28$

30. Van den Berg EK, Schmitz JM, Benedict CR, Malloy CR, Willerson JT, Dehmer J. Transcardiac serotonin concentration is increased in selected patients with limiting angina and complex coronary lesion morphology. Circulation. 1989;79:116-124.

31. Puri VK, Verma M, Saxena AK, Shanker K. Platelet serotonergic mechanisms in ischemic heart disease. Thromb Res. 1990;57:445-51.

32. Hirowatari Y, Hara K, Takahashi H. Serotonin: a novel marker for atherosclerotic vascular disease. Rinsho Byori. 2004;52(8):693-703.

33. Hara K, Hirowatari Y, Yoshika M, Komiyama Y, Tsuka Y, Takahashi H. The ratio of plasma to whole-blood serotonin may be a novel marker of atherosclerotic cardiovascular disease. J Lab Clin Med. 2004;144(1):31-7.

34. Vikenes K, Farstad M, Nordrehaug JE. Serotonin is associated with coronary artery disease and cardiac events. Circulation. 1999;100(5):483-9. 